\title{
Stability of Markov processes nonhomogeneous in time
}

\author{
by Marta TYRAn-Kamińska (Katowice)
}

\begin{abstract}
We study the asymptotic behaviour of discrete time processes which are products of time dependent transformations defined on a complete metric space. Our sufficient condition is applied to products of Markov operators corresponding to stochastically perturbed dynamical systems and fractals.
\end{abstract}

0. Introduction. In some analytical models we need to study the asymptotic behaviour of sequences of the form

$$
x_{n}=T_{n} \circ \ldots \circ T_{1} x_{0},
$$

where $T_{i}: X \rightarrow X$ are given transformations from a metric space $X$ into itself and $x_{0} \in X$ is a starting point. The behaviour of the sequence may be quite complicated even in the case when all the transformations $T_{i}$ are contractions. As the simplest example consider constant transformation $T_{i}(x)=a_{i}$ for $x \in X$. Then, of course, $x_{n}=a_{n}$ and the fact that all $T_{i}$ have Lipschitz constant equal to zero is irrelevant.

A. Lasota proposed to study the behaviour of $\left(x_{n}\right)$ under the assumption

$$
\sum_{n=1}^{\infty} \sup _{x \in X} \varrho\left(T_{n}(x), T_{n+1}(x)\right)<\infty .
$$

We show that in the case when all $T_{i}$ are contractive some more restrictive condition (see (1.2)) is sufficient for the convergence of $\left(x_{n}\right)$. In the specific case when all $T_{i}$ are contractive with the same constant smaller than 1 , our condition reduces to $(0.2)$.

The plan of the paper is as follows. In Section 1 we formulate theorems on asymptotic properties of sequences of the form (0.1) and give some remarks. The proof of the main result is given in Section 2. Section 3 contains basic notions and facts concerning Markov operators acting on measures. Finally,

1991 Mathematics Subject Classification: Primary 60J05; Secondary 47H10, 58F10. Key words and phrases: asymptotic stability, Markov operator, dynamical system. 
in Section 4 we apply our theorem to stochastically perturbed systems and iterated function systems (related to fractals).

1. The convergence theorem. Let $(E, d)$ be an arbitrary metric space. We call a mapping $T: E \rightarrow E$ nonexpansive with respect to the metric $d$ if it satisfies

$$
d(T(u), T(v)) \leq d(u, v) \quad \text { for } u, v \in E,
$$

and $\lambda$-contractive with respect to the metric $d$ if $\lambda \in[0,1)$ and

$$
d(T(u), T(v)) \leq \lambda d(u, v) \quad \text { for } u, v \in E .
$$

As usual, by $T^{n}$ we denote the $n$th iterate of $T$. The set of all positive integers is denoted by $\mathbb{N}$.

Our goal is to study a family $T(n, m)(n \geq m, n, m \in \mathbb{N})$ of transformations from $E$ into itself. We call a family $\{T(n, m)\}$ a process if $T(m, m)=\mathrm{Id}$ (the identity transformation) and

$$
T(n, m)=T(n, k) T(k, m) \quad \text { for } n \geq k \geq m .
$$

Observe that in view of the above condition, a family $\{T(n, m)\}$ is a process if and only if there is a sequence $\left(T_{n}\right)_{n \in \mathbb{N}}$ of transformations such that

$$
T(n, m)=T_{n-1} \circ \ldots \circ T_{m} \quad \text { for } n>m, n, m \in \mathbb{N} .
$$

When $T(n, m)$ is generated by one transformation $T: E \rightarrow E$, then

$$
T(n, m)=T^{n-m}, \quad n \geq m\left(T^{0}=\mathrm{Id}\right) .
$$

We call a process $\{T(n, m)\}$ asymptotically stable if there exists a unique element $u_{*} \in E$ such that

$$
\lim _{n \rightarrow \infty} d\left(T(n, m) v, u_{*}\right)=0 \quad \text { for all } v \in E \text { and } m \in \mathbb{N} .
$$

Now, we are in a position to state our main result.

Theorem 1. Let $(E, d)$ be a metric space and let $\left(T_{n}\right)_{n \in \mathbb{N}}$ be a sequence of arbitrary transformations from $E$ into itself. Assume that there exists an increasing sequence $\left(n_{k}\right)$ of positive integers and a sequence $\left(\lambda_{k}\right)$ of nonnegative real numbers such that for each $k \in \mathbb{N}$ the transformation $T_{n_{k}}$ is $\lambda_{k}$-contractive and

$$
\lim _{k \rightarrow \infty} \frac{1}{1-\lambda_{k}} \sum_{i=n_{k}}^{\infty} \sup _{u \in E} d\left(T_{i}(u), T_{i+1}(u)\right)=0 .
$$

Then for every $m \in \mathbb{N}$ and $u \in E$ we have:

(a) The sequence $(T(n, m)(u))_{n \geq m}$ is Cauchy.

(b) $\lim _{n \rightarrow \infty} d(T(n, m)(u), T(n, m)(v))=0$ for all $v \in E$.

If $(E, d)$ is in addition complete then the process $\{T(n, m)\}$ is asymptotically stable. 
The proof will be given in the next section. Now we discuss some problems related to condition (1.2), which is a key assumption in Theorem 1.

REMARK 1. First observe that if the sequence $\left(\lambda_{k}\right)_{k \in \mathbb{N}}$ tends to a constant $\lambda<1$ or is bounded by a constant $\lambda<1$ then condition (1.2) is equivalent to

$$
\lim _{k \rightarrow \infty} \sum_{i=n_{k}}^{\infty} \sup _{u \in E} d\left(T_{i}(u), T_{i+1}(u)\right)=0 .
$$

REMARK 2. It is worth pointing out that even in the case of a compact metric space assumption (1.2) of Theorem 1 cannot be replaced by condition (1.3) without additional assumptions concerning the transformations $T_{n}$. Consider the following example. Take $E=[0,1]$. Let $T_{n}$ be the identity transformation for odd positive integers $n$, whereas for even $n$ set $T_{n}(u)=$ $\left(1-1 / n^{2}\right) u, u \in E$. Take $n_{k}=2 k, k \in \mathbb{N}$. Then $T_{2 k}$ is $\lambda_{k}$-contractive with $\lambda_{k}=1-1 /\left(4 k^{2}\right)$. Note that $\sup _{u \in E} d\left(T_{i}(u), T_{i+1}(u)\right) \leq 1 / i^{2}$ for every $i \in \mathbb{N}$, hence (1.3) holds. It is easy to calculate that $T(n, 1)(u)=T(n, 2) u=a_{k} u$ for $2 k \leq n<2 k+2$, where

$$
a_{k}=\prod_{i=1}^{k}\left(1-\frac{1}{4 i^{2}}\right) \quad \text { for } k \in \mathbb{N} .
$$

Since the sequence $\left(a_{k}\right)$ tends to $2 / \pi$ as $k \rightarrow \infty$, we have

$$
\lim _{n \rightarrow \infty} T(n, 1)(u)=\frac{2}{\pi} u
$$

and the limit depends on $u$, so the process is not asymptotically stable.

The following theorem shows that the assumptions of Theorem 1 can be modified in a way that will be useful later.

Theorem 2. Let $(E, d)$ be a complete metric space and, for every $n \in \mathbb{N}$, the mapping $T_{n}: E \rightarrow E$ be a nonexpansive transformation with respect to the metric d. Assume that there is a subset $E_{0} \subset E$ and a metric $d_{0}: E_{0} \times E_{0} \rightarrow \mathbb{R}_{+}$such that

(i) $E_{0}$ is dense in $E$ with respect to the metric $d$ and invariant under every $T_{n}$, i.e. $T_{n}\left(E_{0}\right) \subset E_{0}$ for $n \in \mathbb{N}$;

(ii) $d_{0}$ is stronger than d, i.e.

$$
d(u, v) \leq d_{0}(u, v) \quad \text { for } u, v \in E_{0} .
$$

Assume, moreover, that there exists an increasing sequence $\left(n_{k}\right)$ of positive integers and a sequence $\left(\lambda_{k}\right)$ of nonnegative real numbers so that 
(iii) $\lim _{k \rightarrow \infty} \frac{1}{1-\lambda_{k}} \sum_{i=n_{k}}^{\infty} \sup _{u \in E_{0}} d_{0}\left(T_{i}(u), T_{i+1}(u)\right)=0$;

(iv) for each $k \in \mathbb{N}$ the transformation $T_{n_{k}}$ restricted to $E_{0}$ is $\lambda_{k}$-contractive with respect to the metric $d_{0}$.

Under the above assumptions the process $\{T(n, m)\}$ is asymptotically stable and the unique element $u_{*} \in E$ described by condition (1.1) is such that the sequence $\left(T_{n}\left(u_{*}\right)\right)$ tends to $u_{*}$.

Proof. By conditions (iii), (iv) and $T_{n}$-invariance of $E_{0}$ we can use Theorem 1 for $\left(E_{0}, d_{0}\right)$. From Theorem 1(b) and assumption (ii) it follows that for each $m \in \mathbb{N}$ we have

$$
\lim _{n \rightarrow \infty} d(T(n, m)(u), T(n, m)(v))=0 \quad \text { for all } u, v \in E_{0} .
$$

Since $E_{0}$ is dense in $(E, d)$ and each $T_{n}$ is nonexpansive with respect to $d$, (1.4) remains true for $u, v \in E$. The properties (a) and (ii) imply that for every $m \in \mathbb{N}$ and $u \in E_{0}$ the sequence $(T(n, m)(u))$ is also Cauchy with respect to the metric $d$, thus it is convergent in $(E, d)$.

By what we have just shown, for each $m \in \mathbb{N}$ there exists exactly one point, say $u_{m}$, such that

$$
\lim _{n \rightarrow \infty} d\left(T(n, m) v, u_{m}\right)=0 \quad \text { for all } v \in E .
$$

Fix an integer $m \geq 2$ and $u \in E$. Substituting $v=T(m, 1)(u)$ into (1.5) we get

$$
\lim _{n \rightarrow \infty} d\left(T(n, m) T(m, 1)(u), u_{m}\right)=0 .
$$

On the other hand, the sequence $(T(n, 1)(u))$ tends to $u_{1}$. Since for each $n$ sufficiently large $T(n, m) T(m, 1) u=T(n, 1) u$ and this sequence has exactly one limit point, $u_{m}$ must be $u_{1}$. Moreover, by nonexpansiveness of $T_{n}$,

$$
d\left(T_{n+1}\left(u_{1}\right), u_{1}\right) \leq d\left(u_{1}, T(n+1,1)(u)\right)+d\left(T(n, 1)\left(u_{1}\right), u_{1}\right) \quad \text { for } n \in \mathbb{N} .
$$

From (1.5) it now follows that the sequence $\left(T_{n}\left(u_{1}\right)\right)$ tends to $u_{1}$.

Now consider a special case when every transformation is independent of $n$, i.e. $T_{n}=T$. Then obviously condition (iii) is satisfied and we have the following corollary, which was stated by A. Lasota [6].

Corollary 1. Assume that a mapping $T: E \rightarrow E$ defined on a complete metric space is nonexpansive. Suppose there is a subset $E_{0} \subset E$ and a metric $d_{0}: E_{0} \times E_{0} \rightarrow \mathbb{R}_{+}$such that

$\left(\mathrm{i}^{\prime}\right) E_{0}$ is dense in $E$ with respect to the metric $d$ and $T$-invariant;

(ii') $d_{0}$ is stronger than $d$;

(iii') the transformation $T$ restricted to $E_{0}$ is $\lambda$-contractive with respect to the metric $d_{0}$, where $\lambda<1$ is a constant. 
Then $T$ has a unique fixed point $u_{*}$ in $E$ and

$$
\lim _{n \rightarrow \infty} d\left(T^{n}(u), u_{*}\right)=0 \quad \text { for all } u \in E .
$$

2. Proof of Theorem 1. We precede the proof of Theorem 1 with the following lemmas.

Lemma 1. Let $(E, d)$ be a metric space. Assume that a sequence $\left(z_{n}\right)_{n \in \mathbb{N}}$ in $E$ has the following property: that

(I) For every $\varepsilon>0$ there exists a Cauchy sequence $\left(v_{n}\right)_{n \in \mathbb{N}}$ in $E$ such

$$
\limsup _{n \rightarrow \infty} d\left(v_{n}, z_{n}\right) \leq \varepsilon .
$$

Then the sequence $\left(z_{n}\right)$ is Cauchy in $(E, d)$.

The proof of the above lemma is a straightforward consequence of condition (I).

Lemma 2. Let $(E, d)$ be a metric space and $T_{n}, n \in \mathbb{N}$, be arbitrary transformations from $E$ into itself. If there exists $a k \in \mathbb{N}$ and a nonnegative real number $a_{k}$ so that

$$
d\left(T_{k}(u), T_{k}(v)\right) \leq a_{k} d(u, v) \quad \text { for all } u, v \in E,
$$

then for every $z \in E$ and $n>k, n \in \mathbb{N}$,

$$
\begin{aligned}
d(T(n+1, k+1)(z), & \left.T_{k}^{n-k}(z)\right) \\
& \leq \sum_{i=k}^{n-1} \varepsilon_{i}+a_{k} d\left(T(n, k+1)(z), T_{k}^{n-k-1}(z)\right),
\end{aligned}
$$

where

$$
\varepsilon_{i}=\sup _{u \in E} d\left(T_{i}(u), T_{i+1}(u)\right) \quad \text { for } i \in \mathbb{N} .
$$

Proof. Let $z \in E$. For each fixed $n>k$ define $y_{n}=T(n+1, k+1)(z)$ and $x_{n}=T_{k}^{n-k}(z)$. Observe that, according to the recurrent formulas $y_{n}=T_{n}\left(y_{n-1}\right)$ and $x_{n}=T_{k}\left(x_{n-1}\right)$, we have

$$
d\left(y_{n}, x_{n}\right) \leq \sum_{i=k}^{n-1} d\left(T_{i}\left(y_{n-1}\right), T_{i+1}\left(y_{n-1}\right)\right)+d\left(T_{k}\left(y_{n-1}\right), T_{k}\left(x_{n-1}\right)\right) .
$$

From this and assumption (2.1) it follows that

$$
d\left(y_{n}, x_{n}\right) \leq \sum_{i=k}^{n-1} \varepsilon_{i}+a_{k} d\left(y_{n-1}, x_{n-1}\right)
$$

where $\varepsilon_{i}$ are given by (2.3). The last inequality is equivalent to (2.2). 
Proof of Theorem 1. Fix a positive integer $m$. We begin by showing that for every $\varepsilon>0$ there exists $k=k(\varepsilon, m) \in \mathbb{N}$ such that

$$
\limsup _{n \rightarrow \infty} d\left(T(n, m)(u), v_{n}(u)\right) \leq \varepsilon \quad \text { for all } u \in E,
$$

where $v_{n}(u)=T_{n_{k}}^{n-1-n_{k}}\left(T\left(n_{k}+1, m\right)(u)\right)$ for $n>n_{k}$.

Given $\varepsilon>0$, by assumption (1.2) we can choose $k_{0}$ so that

$$
\frac{1}{1-\lambda_{k}} \sum_{i=n_{k}}^{\infty} \varepsilon_{i}<\varepsilon \quad \text { for } k \geq k_{0}
$$

where

$$
\varepsilon_{i}=\sup _{u \in E} d\left(T_{i}(u), T_{i+1}(u)\right) \quad \text { for } i \in \mathbb{N} .
$$

Let $k$ be an integer such that $n_{k}>\max \left\{m, n_{k_{0}}\right\}$ and let $u \in E$. Applying Lemma 2 to the transformation $T_{n_{k}}$ we infer that inequality (2.2) is valid for every $n>n_{k}$ and $z \in E$. In particular, for $z=T\left(n_{k}+1, m\right)(u)$ and $n>n_{k}$ we obtain

$$
\begin{aligned}
& d\left(T\left(n+1, n_{k}+1\right)\left(T\left(n_{k}+1, m\right)(u)\right), T_{n_{k}}^{n-k}\left(T\left(n_{k}+1, m\right)(u)\right)\right) \\
& \leq \sum_{i=n_{k}}^{n-1} \varepsilon_{i}+\lambda_{k} d\left(T\left(n, n_{k}+1\right)\left(T\left(n_{k}+1, m\right)(u)\right), T_{n_{k}}^{n-1-k}\left(T\left(n_{k}+1, m\right)(u)\right)\right) .
\end{aligned}
$$

This estimate and (2.5) imply that

$$
\begin{aligned}
d\left(T(n+1, m)(u), v_{n+1}(u)\right) & \leq\left(1-\lambda_{k}\right) \varepsilon+\lambda_{k} d\left(T(n, m)(u), v_{n}(u)\right), \\
\text { where } \quad v_{n}(u) & =T_{n_{k}}^{n-1-n_{k}}\left(T\left(n_{k}+1, m\right)(u)\right) \text { for } n>n_{k} .
\end{aligned}
$$

It follows that the numerical sequence $\left(d\left(T(n, m)(u), v_{n}(u)\right)\right)_{n>n_{k}}$ is bounded and that

$$
\begin{aligned}
\limsup _{n \rightarrow \infty} d(T(n+1, m)(u) & \left., v_{n+1}(u)\right) \\
\leq & \left(1-\lambda_{k}\right) \varepsilon+\lambda_{k} \limsup _{n \rightarrow \infty} d\left(T(n, m)(u), v_{n}(u)\right) .
\end{aligned}
$$

Consequently,

$$
\limsup _{n \rightarrow \infty} d\left(T(n, m)(u), v_{n}(u)\right) \leq \varepsilon,
$$

which completes the proof of (2.4).

Since for each $k \in \mathbb{N}$ the transformation $T_{n_{k}}$ is $\lambda_{k}$-contractive, the sequence $\left(T_{n_{k}}^{n-n_{k}}(z)\right)_{n \geq n_{k}}$ is Cauchy for $z \in E$. From this and (2.4) it follows that for every $u \in E$ the sequence $(T(n, m)(u))_{n \geq m}$ satisfies condition (I) of Lemma 1, so the proof of (a) is complete. 
To prove (b) fix $\varepsilon>0$ and choose $k$ such that (2.4) holds. Let $u, v \in E$. Clearly,

$$
\begin{aligned}
d(T(n, m)(u), T(n, m)(v)) \leq & d\left(T(n, m)(u), T_{n_{k}}^{n-1-n_{k}}\left(T\left(n_{k}+1, m\right)(u)\right)\right) \\
& +d\left(T(n, m)(v), T_{n_{k}}^{n-1-n_{k}}\left(T\left(n_{k}+1, m\right)(v)\right)\right) \\
& +\lambda_{k}^{n-1-n_{k}} d\left(T\left(n_{k}+1, m\right)(u), T\left(n_{k}+1, m\right)(v)\right)
\end{aligned}
$$

for all $n>n_{k}$. By assumption, $\lambda_{k}<1$, therefore the last term on the righthand side converges to zero as $n \rightarrow \infty$. Hence and from (2.4) we obtain

$$
\limsup _{n \rightarrow \infty} d(T(n, m)(u), T(n, m)(v))<2 \varepsilon .
$$

Since $\varepsilon>0$ is arbitrary, this completes the proof of (b).

The second part of the theorem is obvious.

3. Markov operators. Let $(X, \varrho)$ be a Polish space, i.e. a separable complete metric space. We denote by $\mathcal{B}_{X}$ the $\sigma$-algebra of Borel subsets of $X$. The space of all finite Borel measures (nonnegative, $\sigma$-additive) on $X$ will be denoted by $\mathcal{M}$. The subspace of $\mathcal{M}$ which contains only normalized measures (i.e. $\mu(X)=1, \mu \in \mathcal{M}$ ) will be denoted by $\mathcal{M}_{1}$ and its elements will be called distributions. Furthermore,

$$
\mathcal{M}_{\text {sig }}=\left\{\mu_{1}-\mu_{2}: \mu_{1}, \mu_{2} \in \mathcal{M}\right\}
$$

denotes the space of finite signed measures.

As usual, we denote by $B(X)$ the space of all bounded Borel measurable functions $f: X \rightarrow \mathbb{R}$ and by $C(X)$ its subspace containing all continuous functions. Both spaces are considered with the norm

$$
\|f\|=\sup _{x \in X}|f(x)| .
$$

For $f \in B(X)$ and $\mu \in \mathcal{M}_{\text {sig }}$ we write

$$
\langle f, \mu\rangle=\int_{X} f(x) \mu(d x) .
$$

The space $\mathcal{M}_{\text {sig }}$ is a normed vector space with the Fortet-Mourier norm $([3],[9])$

where

$$
\|\mu\|_{\mathcal{F}}=\sup \{|\langle f, \mu\rangle|: f \in \mathcal{F}\} \quad \text { for } \mu \in \mathcal{M}_{\text {sig }}
$$

$$
\mathcal{F}=\{f \in C(X):\|f\| \leq 1 \text { and }|f(x)-f(y)| \leq \varrho(x, y) \text { for } x, y \in X\} .
$$

In general, $\left(\mathcal{M}_{\text {sig }},\|\cdot\|_{\mathcal{F}}\right)$ is not a complete space. However, it is known that the set $\mathcal{M}_{1}$ with the distance $\left\|\mu_{1}-\mu_{2}\right\|_{\mathcal{F}}$ is a complete metric space $([9])$ and the convergence

$$
\lim _{n \rightarrow \infty}\left\|\mu_{n}-\mu\right\|_{\mathcal{F}}=0 \quad \text { for } \mu_{n}, \mu \in \mathcal{M}_{1}
$$


is equivalent to weak convergence of distributions defined by

$$
\lim _{n \rightarrow \infty}\left\langle f, \mu_{n}\right\rangle=\langle f, \mu\rangle \quad \text { for all } f \in C(X) .
$$

In $\mathcal{M}_{1}$ we introduce another distance, the Hutchinson metric ([5], [6]):

$$
\left\|\mu_{1}-\mu_{2}\right\|_{\mathcal{H}}=\sup \left\{\left|\left\langle f, \mu_{1}-\mu_{2}\right\rangle\right|: f \in \mathcal{H}\right\} \quad \text { for } \mu_{1}, \mu_{2} \in \mathcal{M}_{1},
$$

where

$$
\mathcal{H}=\{f \in C(X):|f(x)-f(y)| \leq \varrho(x, y) \text { for } x, y \in X\} ;
$$

$\left\|\mu_{1}-\mu_{2}\right\|_{\mathcal{H}}$ is always defined but for some $\mu_{1}, \mu_{2} \in \mathcal{M}_{1}$ it may be infinite. Note that, because of the inclusion $\mathcal{F} \subset \mathcal{H}$, we always have

$$
\left\|\mu_{1}-\mu_{2}\right\|_{\mathcal{F}} \leq\left\|\mu_{1}-\mu_{2}\right\|_{\mathcal{H}} \quad \text { for } \mu_{1}, \mu_{2} \in \mathcal{M}_{1} \text {. }
$$

A linear mapping $P: \mathcal{M}_{\text {sig }} \rightarrow \mathcal{M}_{\text {sig }}$ is called a Markov operator if $P\left(\mathcal{M}_{1}\right) \subset \mathcal{M}_{1}$ (see $\left.[6,7,9]\right)$. Now we will show how to construct a Markov operator.

Let a linear operator $U: B(X) \rightarrow B(X)$ be given. Assume that $U$ satisfies the following conditions:

(U1) $U f \geq 0$ for $f \in B(X), f \geq 0$;

(U2) $U 1_{X}=1_{X}$;

(U3) if a nonincreasing sequence $\left(f_{n}\right)_{n \in \mathbb{N}}$ in $B(X)$ is pointwise convergent to 0 then

$$
\lim _{n \rightarrow \infty} U f_{n}(x)=0 \quad \text { for } x \in X ;
$$

(U4) $U f \in C(X)$ for $f \in C(X)$.

Define an operator $P: \mathcal{M}_{\text {sig }} \rightarrow \mathcal{M}_{\text {sig }}$ by

$$
P \mu(A)=\left\langle U 1_{A}, \mu\right\rangle \quad \text { for } A \in \mathcal{B}_{X}, \mu \in \mathcal{M}_{\text {sig }} .
$$

It can be easily shown (see [6]) that $P$ is the unique Markov operator satisfying

$$
\langle U f, \mu\rangle=\langle f, P \mu\rangle \quad \text { for } f \in B(X), \mu \in \mathcal{M}_{\text {sig }},
$$

so $U$ is the dual operator to $P$. In particular, substituting $\mu=\delta_{x}$ into (3.2) we obtain

$$
U f(x)=\left\langle f, P \delta_{x}\right\rangle \quad \text { for } x \in X, f \in B(X),
$$

where $\delta_{x} \in \mathcal{M}_{1}$ is the point (Dirac) unit measure supported at $x$.

We call $P$ a Feller operator if its dual operator $U$ satisfies condition (U4).

Finally, for convenience, we present some facts concerning Markov operators which we need in the sequel (see [6]).

Proposition 1. Let $P: \mathcal{M}_{\text {sig }} \rightarrow \mathcal{M}_{\text {sig }}$ be a Feller operator and let its dual operator $U$ satisfy

$$
|U f(x)-U f(\bar{x})| \leq \lambda \varrho(x, \bar{x}) \quad \text { for } x, \bar{x} \in X \text { and } f \in \mathcal{H},
$$


where $\lambda \leq 1$ is a nonnegative constant. Then $P$ is nonexpansive with respect to the Fortet-Mourier norm and

$$
\left\|P \mu_{1}-P \mu_{2}\right\|_{\mathcal{H}} \leq \lambda\left\|\mu_{1}-\mu_{2}\right\|_{\mathcal{H}} \quad \text { for } \mu_{1}, \mu_{2} \in \mathcal{M}_{1} .
$$

If, moreover, there is a measure $\nu \in \mathcal{M}_{1}$ such that

$$
\|P \nu-\nu\|_{\mathcal{H}}<\infty,
$$

then $\mathcal{M}_{0}=\left\{\mu \in \mathcal{M}_{1}:\|\mu-\nu\|_{\mathcal{H}}<\infty\right\}$ is a dense and P-invariant subset of the metric space $\left(\mathcal{M}_{1},\|\cdot\|_{\mathcal{F}}\right)$, and it is a metric space when equipped with the Hutchinson metric.

4. Dynamical systems. Throughout this section $(X, \varrho)$ is a Polish space and $(I, \mathcal{A})$ is a measurable space. We consider dynamical systems in a general form (for the homogeneous cases see $[7-8,10]$ ). Let $(\Omega, \Sigma$, prob) be a probability space and let $\eta_{n}: \Omega \rightarrow I, n \in \mathbb{N}$, be a sequence of independent random elements (measurable transformations) having the same distribution, i.e. the measure

$$
\psi(A)=\operatorname{prob}\left(\eta_{n} \in A\right) \quad \text { for } A \in \mathcal{A}
$$

is the same for all $n$. Assume that for each $n \in \mathbb{N}$ a measurable transformation $S_{n}: X \times I \rightarrow X$ is given.

Consider a sequence $\xi_{n}: \Omega \rightarrow X$ of random elements defined by the recurrent formula

$$
\xi_{n}=S_{n}\left(\xi_{n-1}, \eta_{n}\right) \quad \text { for } n \in \mathbb{N},
$$

where the initial value $\xi_{0}: \Omega \rightarrow X$ is a random element independent of the sequence $\left(\eta_{n}\right)$.

We make the following assumptions:

(A1) For each $n$ there exists a measurable function $L_{n}: I \rightarrow \mathbb{R}_{+}$such that

$$
\varrho\left(S_{n}(x, y), S_{n}(\bar{x}, y)\right) \leq L_{n}(y) \varrho(x, \bar{x}) \quad \text { for } x, \bar{x} \in X, y \in I
$$

and

$$
a_{n}=\int_{I} L_{n}(y) \psi(d y) \leq 1 .
$$

(A2) There exists a point $x_{0} \in X$ such that

$$
b_{n}=\int_{I} \varrho\left(x_{0}, S_{n}\left(x_{0}, y\right)\right) \psi(d y)<\infty \quad \text { for } n \in \mathbb{N} .
$$

(A3) There exists an increasing sequence $\left(n_{k}\right)_{k \in \mathbb{N}}$ of integers so that 
$a_{n_{k}}<1$ for $k \in \mathbb{N}$, and

$$
\lim _{k \rightarrow \infty} \frac{1}{1-a_{n_{k}}} \sum_{i=n_{k}}^{\infty} \sup _{x \in X} \int_{I} \varrho\left(S_{i}(x, y), S_{i+1}(x, y)\right) \psi(d y)=0 .
$$

The sequence given by (4.1) is a Markov process for which the onestep transition function may depend on $n$. We now give a rule on how the distributions of $\xi_{n}$ evolve in time by means of Markov operators. For each integer $n$ define an operator $U_{n}$ acting on $B(X)$ by setting

$$
U_{n} f(x)=\int_{I} f\left(S_{n}(x, y)\right) \psi(d y) \quad \text { for } x \in X, f \in B(X) .
$$

Of course, $U_{n}: B(X) \rightarrow B(X)$ is a linear operator satisfying (U1)-(U3). Moreover, from (4.2) it follows that for every $y \in I$ the transformation $S_{n}(\cdot, y): X \rightarrow X$ is continuous, therefore $U_{n} f \in C(X)$ for $f \in C(X)$. Hence, according to (3.1), the Markov operator $P_{n}$ is of the form

$$
P_{n} \mu(A)=\int_{X}\left\{\int_{I} 1_{A}\left(S_{n}(x, y)\right) \psi(d y)\right\} \mu(d x) \quad \text { for } A \in \mathcal{B}_{X}, \mu \in \mathcal{M}_{\text {sig. }} .
$$

We are interested in the asymptotic behaviour of the distributions

$$
\mu_{n}(A)=\operatorname{prob}\left(\xi_{n} \in A\right) \quad \text { for } A \in \mathcal{B}_{X}, n=0,1,2, \ldots,
$$

where $\left(\xi_{n}\right)$ is defined by (4.1). Using the form of $P_{n}$ it is easy to check (see [7]) that

$$
\mu_{n}=P_{n} \mu_{n-1} \quad \text { for } n \in \mathbb{N} .
$$

Consequently, $\mu_{n}=P(n+1,1) \mu_{0}, n \in \mathbb{N}$.

Now, using Theorem 2 we can prove the main result of this section, which is a nonhomogeneous (in time) version of a result due to A. Lasota and M. C. Mackey [7] (p. 423).

Theorem 3. Assume that the sequence $\left(S_{n}\right)$ satisfies (A1)-(A3). Then there exists a unique measure $\mu_{*} \in \mathcal{M}_{1}$ such that $\lim _{n \rightarrow \infty}\left\|P_{n} \mu_{*}-\mu_{*}\right\|_{\mathcal{F}}=0$ and

$$
\lim _{n \rightarrow \infty}\left\|P(n, m) \mu-\mu_{*}\right\|_{\mathcal{F}}=0 \quad \text { for all } \mu \in \mathcal{M}_{1}, m \in \mathbb{N} .
$$

Proof. We show that the Markov operators $P_{n}: \mathcal{M}_{1} \rightarrow \mathcal{M}_{1}, n \in \mathbb{N}$, satisfy the requirements of Theorem 2 . Fix $n$. It is easy to calculate that, in view of (4.4) and (A1),

$$
\left|U_{n} f(x)-U_{n} f(\bar{x})\right| \leq a_{n} \varrho(x, \bar{x}) \quad \text { for } x, \bar{x} \in X \text { and } f \in \mathcal{H},
$$

where, according to (4.3), $a_{n} \leq 1$. Now, we are going to verify that

$$
\left\|P_{n} \delta_{x_{0}}-\delta_{x_{0}}\right\|_{\mathcal{H}} \leq b_{n},
$$

where $x_{0}$ and $b_{n}$ are described in (A2). Indeed, if $f \in \mathcal{H}$ then $\left|\left\langle f, P_{n} \delta_{x_{0}}-\delta_{x_{0}}\right\rangle\right|$ $=\left|U_{n} f\left(x_{0}\right)-f\left(x_{0}\right)\right|$. Since $\psi(I)=1$, we have $f\left(x_{0}\right)=\int_{I} f\left(x_{0}\right) \psi(d y)$, and 
consequently,

$$
\left|\left\langle f, P_{n} \delta_{x_{0}}-\delta_{x_{0}}\right\rangle\right| \leq \int_{I} \varrho\left(S_{n}\left(x_{0}, y\right), x_{0}\right) \psi(d y) .
$$

The right-hand side does not depend on $f$, hence the desired estimate follows. Thus, by Proposition 1 the Markov operator $P_{n}$ is nonexpansive with respect to the Fortet-Mourier metric and the metric space $\left(\mathcal{M}_{0},\|\cdot\|_{\mathcal{H}}\right)$ satisfies condition (i) of Theorem 2, where

$$
\mathcal{M}_{0}=\left\{\mu \in \mathcal{M}_{1}:\left\|\mu-\delta_{x_{0}}\right\|_{\mathcal{H}}<\infty\right\} .
$$

Moreover, by (3.3) we have $\left\|P_{n} \mu_{1}-P_{n} \mu_{2}\right\|_{\mathcal{H}} \leq a_{n}\left\|\mu_{1}-\mu_{2}\right\|_{\mathcal{H}}$ for all $n$, and $a_{n_{k}}<1$ for all $k \in \mathbb{N}$ by (A3), therefore condition (iv) is satisfied as well.

It remains to verify (iii). Observe that for $f \in \mathcal{H}$ and $\mu \in \mathcal{M}_{0}$ we have $\left|\left\langle f, P_{n} \mu-P_{n+1} \mu\right\rangle\right|=\left|\left\langle U_{n} f-U_{n+1} f, \mu\right\rangle\right| \leq\left\|U_{n} f-U_{n+1} f\right\|$ for all $n \in \mathbb{N}$. The last term can be estimated as follows:

$$
\begin{aligned}
\left\|U_{n} f-U_{n+1} f\right\| & \leq \sup _{x \in X} \int_{I}\left|f\left(S_{n}(x, y)\right)-f\left(S_{n+1}(x, y)\right)\right| \psi(d y) \\
& \leq \sup _{x \in X} \int_{I} \varrho\left(S_{n}(x, y), S_{n+1}(x, y)\right) \psi(d y) .
\end{aligned}
$$

The right-hand side does not depend on $f \in \mathcal{H}$ and $\mu \in \mathcal{M}_{0}$, thus

$$
\sup _{\mu \in \mathcal{M}_{0}}\left\|P_{n} \mu-P_{n+1} \mu\right\|_{\mathcal{H}} \leq \sup _{x \in X} \int_{I} \varrho\left(S_{n}(x, y), S_{n+1}(x, y)\right) \psi(d y),
$$

which, according to (A3), proves condition (iii). Consequently, making use of Theorem 2 completes the proof.

Now, we give some examples of applications of Theorem 3. First, we consider iterated function systems $[1-2,6-8,9,10]$. In our case transformations vary in each step.

ExAmple 1. Let $N$ be a positive integer and for each $n \in \mathbb{N}$ let $S_{i}^{n}: X \rightarrow X, i=1, \ldots, N$, be a sequence of transformations such that

$$
\varrho\left(S_{i}^{n}(x), S_{i}^{n}(\bar{x})\right) \leq L_{i}^{n} \varrho(x, \bar{x}) \quad \text { for } x, \bar{x} \in X .
$$

Moreover, let $p_{i}, i=1, \ldots, N$, be a sequence of positive numbers such that $p_{1}+\ldots+p_{N}=1$. We define a random sequence $\left(\xi_{n}\right)$ in the following way. If an initial point $x_{0}$ is given, we select a transformation $S_{i}^{1}$ with probability $p_{i}$ and define $x_{1}=S_{i}^{1}\left(x_{0}\right)$. Having defined the points $x_{1}, \ldots, x_{n}$ we select a transformation $S_{i}^{n+1}$ with probability $p_{i}$ and define $x_{n+1}=S_{i}^{n+1}\left(x_{n}\right)$. This scheme can be described in terms of the following dynamical system. Let $I=\{1, \ldots, N\}$ and let $\eta_{n}: \Omega \rightarrow I, n \in \mathbb{N}$, be a sequence of independent random variables with $\operatorname{prob}\left(\eta_{n}=i\right)=p_{i}$. Set $S_{n}(x, i)=S_{i}^{n}(x)$ for $x \in X$, $i \in I, n \in \mathbb{N}$. 
If we assume that $a_{n}=\sum_{i=1}^{N} p_{i} L_{i}^{n} \leq 1$ for $n \in \mathbb{N}$, $\liminf _{n \rightarrow \infty} a_{n}<1$, and the series $\sum_{n=1}^{\infty} \sup _{x \in X} \varrho\left(S_{i}^{n}(x), S_{i}^{n+1}(x)\right)$ is convergent for each $i \in I$, then all the assumptions of Theorem 3 are satisfied. Thus, the process $\{P(n, m)\}$ generated by the Markov operators

$$
P_{n} \mu(A)=\sum_{i=1}^{N} p_{i} \mu\left(\left(S_{i}^{n}\right)^{-1}(A)\right) \quad \text { for } A \in \mathcal{B}_{X}, \mu \in \mathcal{M}_{1}, n \in \mathbb{N}
$$

is asymptotically stable.

The next example concerns dynamical systems with multiplicative perturbations $[4,11]$.

ExAmPLE 2. Let $(X,\|\cdot\|)$ be a separable Banach space or a closed cone in such a space and $I=[0, \infty)$. For each $n \in \mathbb{N}$ consider the map $S_{n}: X \times I \rightarrow X$ of the form

$$
S_{n}(x, y)=y T_{n}(x) \quad \text { for } x \in X, y \in I,
$$

where $T_{n}: X \rightarrow X$ satisfies $\left\|T_{n}(x)-T_{n}(\bar{x})\right\| \leq c_{n}\|x-\bar{x}\|$ for $x, \bar{x} \in X$ with a nonnegative constant $c_{n}$. Assume that the first moment of the random variables $\eta_{n}: \Omega \rightarrow I$ is finite, i.e.

$$
\int_{I} y \psi(d y)=K<\infty .
$$

If $c_{n} K \leq 1$ for $n \in \mathbb{N}$, $\liminf _{n \rightarrow \infty} c_{n}<1 / K$ and $\sum_{n=1}^{\infty} \sup _{x \in X} \| T_{n}(x)-$ $T_{n+1}(x) \|$ is convergent, then all the assumptions of Theorem 3 are satisfied. Thus, the process $\{P(n, m)\}$ generated by the Markov operators

$$
P_{n} \mu(A)=\int_{X}\left\{\int_{I} 1_{A}\left(y T_{n}(x)\right) \psi(d y)\right\} \mu(d x) \quad \text { for } A \in \mathcal{B}_{X}, \mu \in \mathcal{M}_{1}, n \in \mathbb{N}
$$

is asymptotically stable.

\section{References}

[1] M. F. Barnsley, Fractals Everywhere, Academic Press, New York, 1988.

[2] M. F. Barnsley, S. G. Demko, J. H. Elton and J. S. Geronimo, Invariant measures arising from iterated function systems with place dependent probabilities, Ann. Inst. Henri Poincaré 24 (1988), 367-394.

[3] R. Fortet et B. Mourier, Convergence de la répartition empirique vers la répartition théorétique, Ann. Sci. École Norm. Sup. 70 (1953), 267-285.

[4] K. Horbacz, Dynamical systems with multiplicative perturbations: the strong convergence of measures, Ann. Polon. Math. 58 (1993), 85-93.

[5] J. Hutchinson, Fractals and self-similarity, Indiana Univ. Math. J. 30 (1981), 713-747. 
[6] A. Lasota, From fractals to stochastic differential equations, in: Chaos-The Interplay Between Stochastic and Deterministic Behaviour (Karpacz '95), Lecture Notes in Phys. 457, Springer, 1995, 235-255.

[7] A. Lasota and M. C. Mackey, Chaos, Fractals, and Noise-Stochastic Aspects of Dynamics, Springer, 1994.

[8] - - - Stochastic perturbation of dynamical systems: the weak convergence of measures, J. Math. Anal. Appl. 138 (1989), 232-248.

[9] A. Lasota and J. Yorke, Lower bound technique for Markov operators and iterated function systems, Random Comput. Dynam. 2 (1994), 41-77.

[10] K. Łoskot and R. Rudnicki, Limit theorems for stochastically perturbed dynamical systems, J. Appl. Probab. 32 (1995), 459-469.

[11] K. Oczkowicz, Asymptotic stability of Markov operators corresponding to the dynamical systems with multiplicative perturbations, Ann. Math. Sil. 7 (1993), 99-108.

Institute of Mathematics

Silesian University

Bankowa 14

40-007 Katowice, Poland

E-mail: mtyran@ux2.math.us.edu.pl

Reçu par la Rédaction le 8.4.1998

Révisé le 13.7.1998 